treatment outcomes. In the future, these sex differences could impact management strategies.

Disclosures E. Roney: None. D. Lukins: None. M. Nisiewicz: None. A. Alhajeri: None. S. Grupke: None. J. Fraser: None.

\section{E-023 ALAGILLE SYNDROME WITH BILATERAL LARGE PARA- OPHTHALMIC ANEURYSMS IN A 14-YEAR-OLD GIRL: TREATMENT STRATEGY AND REVIEW}

${ }^{1} \mathrm{~S}$ Suzuki*, ${ }^{2} \mathrm{~L}$ Hsieh, ${ }^{3} \mathrm{~A}$ Patel, ${ }^{4} \mathrm{M}$ Muhonen. 'Neurological Surgery, Univiersity of California Invine, Orange, $C A ;{ }^{2}$ Hematology, Children's Hospital of Orange County, Orange, $C A$; ${ }^{3}$ Gastroenterology, Children's Hospital of Orange County, Orange, $C A ;{ }^{4}$ Neurological Surgery, Children's Hospital of Orange County, Orange, CA

\subsection{6/neurintsurg-2019-SNIS.98}

Background Alagille syndrome (AGS) is a pediatric multisystem autosomal dominant disorder caused by mutations in the JAG1 and NOTCH 2 genes. The feature of the syndrome is broad-spectrum including bile duct paucity with cholestasis, characteristic facies, and anomalies in the cardiac, renal, skeletal, ocular system. Various intracranial vascular abnormalities have also been reported. We describe a case of AGS with bilateral large unruptured para-ophthalmic aneurysms, which were successfully treated with endovascular therapy.

Subject and methods A 14-year-old girl with genetically diagnosed AGS presented with bifrontal pressure headache. MRI of the brain showed no intracranial hemorrhage. However, MRA revealed bilateral internal carotid artery (ICA) supraclinoid segment aneurysms, which increased in size on the follow-up MRA. A diagnostic cerebral angiogram confirmed large para-ophthalmic aneurysms; $8 \times 9 \times 16 \mathrm{~mm}$ with $6 \mathrm{~mm}$ neck (right), $7 \times 8 \times 12 \mathrm{~mm}$ with $4 \mathrm{~mm}$ neck (left), with dysplastic supraclinoid ICAs and M1 segments (figure A). Therefore, a decision was made to treat the aneurysms with endovascular measures.

Results The treatment team was assembled with pediatric hepatobiliary and hematology services in concerning her underlying liver dysfunction, age, and anticipation of using intracranial stents or flow diversion devices with dual antiplatelet therapy (DAP). Platelet function assay, P2Y12 platelet function, and liver function tests were carefully monitored pre- and post-procedure to adjust the DAP. The right ICA para-ophthalmic aneurysm was treated with Pipeline Embolization Device and detachable coils successfully. Four weeks after, the left ICA para-ophthalmic aneurysm was also treated successfully with the same maneuver. In each treatment, the patient was discharged to her home on the following day without complication. Clopidogrel was discontinued after six months with the continuation of aspirin $81 \mathrm{mg}$ daily. The bifrontal pressure sensation disappeared, and no new symptom has been reported since the treatment. One year follow up angiogram illustrates complete aneurysm dome occlusion and the flow diversion device patency (figure B).

Conclusion To our knowledge, this case is the first report of treated the intracranial aneurysms associated with AGS utilizing flow diversion devices. The growing body of literature describes the intracranial aneurysm and intracranial hemorrhage lead to significant morbidity and mortality in patients AGS. It would be warranted to screen with noninvasive imaging modalities, such as MRA and CTA for the patients with AGS who develop alarming neurovascular symptoms. Further, if indicated, endovascular therapy with multidisciplinary team would be considered in the setting of multisystem disorders such as AGS.

Disclosures S. Suzuki: None. L. Hsieh: None. A. Patel: None. M. Muhonen: None.

\section{E-024 LOW PROFILE TRANSRADIAL PIPELINE EMBOLIZATION}

S Satti ${ }^{*}$, T Eden. Neurointerventional Surgery, Christiana Care Health System, Newark, DE

\subsection{6/neurintsurg-2019-SNIS.99}

Purpose Retrospective review of all Medtronic Pipeline Flex flow diverting stent placement at single center with attention to guiding catheter selection, technical success, intracranial complications, access site complications and length of procedure. Summarize technical advantages and limitations of each access strategy and present a simplified 6 French triaxial system for flow diversion stent placement via a transradial/transulnar approach. We also present some tips and tricks to optimize procedural success including transulnar access and direct radial access.

Materials and methods Single Center retrograde suspect of review of all Medtronic Pipeline Embolization Flex procedures performed between October 2015-March 2019 via arm access (radial or ulnar). Three different $6 \mathrm{~F}$ constructs were used for arm access:

1. Direct radial access with long sheath-Terumo destination slender

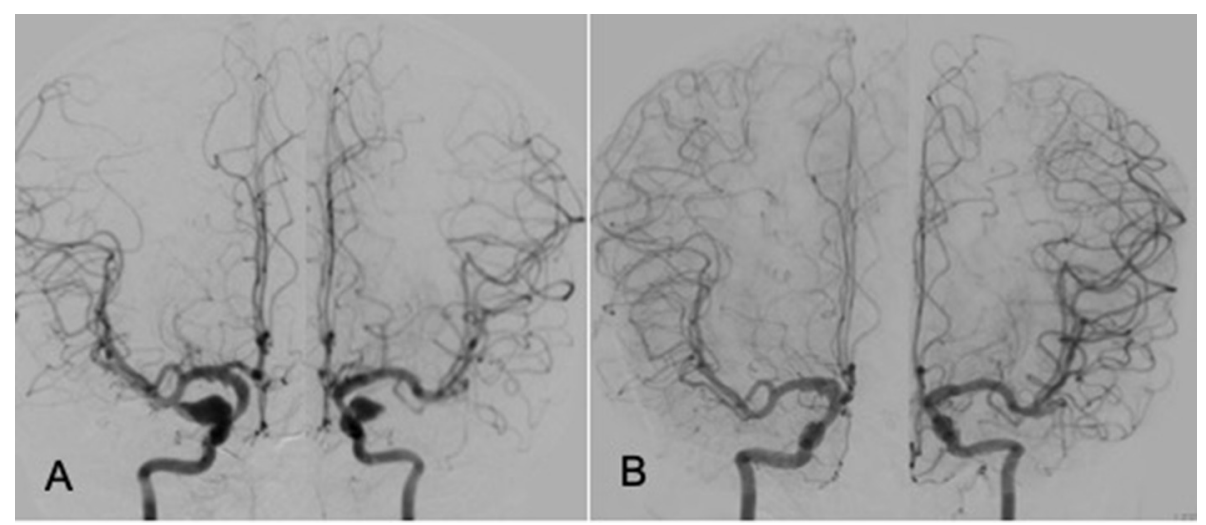

Abstract E-023 Figure 1 